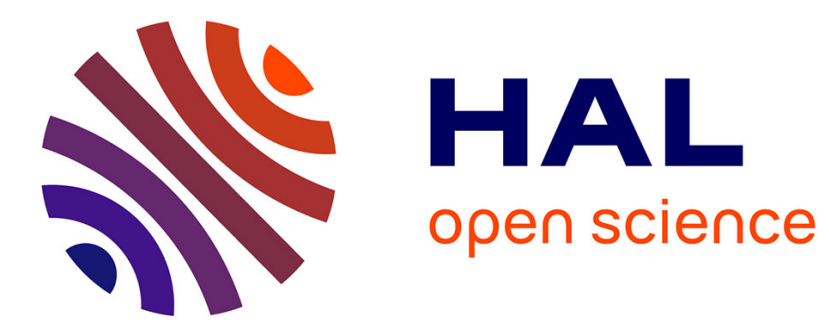

\title{
Homogenization of corrugated core sandwiches panels
}

\author{
Natacha Buannic, Patrice Cartraud, Tanguy Quesnel
}

\section{To cite this version:}

Natacha Buannic, Patrice Cartraud, Tanguy Quesnel. Homogenization of corrugated core sandwiches panels. Composite Structures, 2003, 59 (3), pp.298-311. 10.1016/S0263-8223(02)00246-5 . hal01006930

\section{HAL Id: hal-01006930 \\ https://hal.science/hal-01006930}

Submitted on 21 Nov 2016

HAL is a multi-disciplinary open access archive for the deposit and dissemination of scientific research documents, whether they are published or not. The documents may come from teaching and research institutions in France or abroad, or from public or private research centers.
L'archive ouverte pluridisciplinaire HAL, est destinée au dépôt et à la diffusion de documents scientifiques de niveau recherche, publiés ou non, émanant des établissements d'enseignement et de recherche français ou étrangers, des laboratoires publics ou privés.

\section{(c)(1)}

Distributed under a Creative Commons Attribution| 4.0 International License 


\title{
Homogenization of corrugated core sandwich panels
}

\author{
Natacha Buannic ${ }^{\mathrm{a}}$, Patrice Cartraud ${ }^{\mathrm{b}}$, Tanguy Quesnel \\ a Principia Marine, 1 Rue de la Noe, B.P. 72108, 44321 Nantes Cedex 3, France \\ b Laboratoire Mécanique et Matériaux, Ecole Centrale de Nantes, 1, Rue de la Noe, B.P. 92101, 44321 Nantes Cedex 3, France
}

\begin{abstract}
The present work is devoted to the computation of the effective properties of corrugated core sandwich panels. Due to their periodic structure, the homogenization theory is used, based on the asymptotic expansion method. At the leading order, an equivalent Kirchhoff Love homogeneous plate is derived, with an overall behavior obtained from basic cell problems posed on the three dimensional period of the panel. The finite element computation of these effective properties is presented in this paper. The accuracy of the homogenization method is proved, since the real panel and equivalent plate responses are very close for membrane and pure bending loadings. However, a discrepancy appears for simple bending loading, underlining that transverse shear effects cannot be neglected. Therefore, a specific study is developed in order to derive the transverse shear stiffness, thus enabling to de termine an equivalent Reissner Mindlin homogeneous plate.
\end{abstract}

Keywords: Homogenization; Effective stiffness; Corrugated core; Sandwich panel; Finite element method

\section{Introduction}

Sandwich structural panels are widely used in many industrial areas, and the purpose of the present paper is to investigate their applications in shipbuilding, as an alternative to stiffened panels.

To this aim, a comparative study of different panels will be made with respect to a reference stiffened panel similar to those traditionally used in shipbuilding. The analysis will be limited to their ratios stiffness over mass.

The sandwich panels considered here are corrugated core sandwich panels, the core and the facings being made of steel. Four different core shapes were selected for this study, owing to their easy manufacturing by laser welding. The unidirectional corrugated core is: (a) straight, (b) of hat type, (c) triangular or (d) curvilinear. The stiffened panel (e) used as a reference in the study is also presented in Fig. 1.

Several approaches to the modeling of these structural elements are discussed in the literature, see e.g. the review article [1].
For instance, a three-dimensional (3D) finite element model can be used, where the actual geometry of the core is represented. However, such a model is bound to have a high computational cost, and is inadequate when one is interested in the overall response of the structure. At the scale of a ship, these panels present indeed an overall plate-like behavior, and the problem is then to define the effective plate stiffnesses.

Several authors proposed to use an equivalent continuum instead of the core, and then combine it with a 3D model [2] or a bidimensional plate model [3] (in both references, a honeycomb core is considered. For a variety of cellular core geometries, see the recent review article [4]). In the plate model, the sandwich is then modeled as several layers, and some approximations are made in the direction of the thickness. This type of approach was used among other things for the corrugated cores that are of interest here. Then, from a strength-ofmaterials type of theory, analytical expressions for the characteristics of the equivalent continuum are given in $[5,6]$. Different geometries of corrugation are studied in the latter reference.

Another approach consists in using the homogenization theory, taking benefit from the periodicity exhibited by the structure in its plane. Consequently, the basic cell is made of the core and the facings, i.e. the core is not considered independently. At this stage, it is 


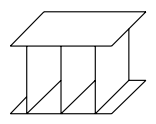

(a) Straight

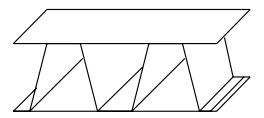

(b) Hat type

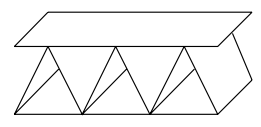

(c) Triangular

Fig. 1. Core shapes of sandwich panels of interest and traditional stiffened panel.

noteworthy that the panels studied here are thin 3D structures with periodic microstructure in direction 1 , and invariant in direction 2 of plane $\left(x_{1}, x_{2}\right)$. These panels are therefore a special case of panels with periodic components in both directions of the plane. Such structures are characterized by the existence of two small parameters noted $e$ and $\varepsilon: e$ is the ratio between panel thickness $t$ and a characteristic dimension $L$ of the panel in its plane, and $\varepsilon$ is the ratio between period length $X_{1}$ or $X_{2}$ and $L$ (see Fig. 2).

The purpose of homogenization is then to substitute the thin initial heterogeneous structure with an equivalent homogeneous plate. This leads to making the two small parameters of the problem tend to zero. This approach was studied theoretically by Caillerie [7], where it was shown that depending on the order in which the two small parameters tend to zero, namely, one after the other or both simultaneously, varied results can be obtained for the effective stiffnesses. Therefore, several homogenization methods are available, with their own domain of validity. Our objective here is not to discuss these different methods, but its interesting to note that replacing the core by an equivalent medium amounts to

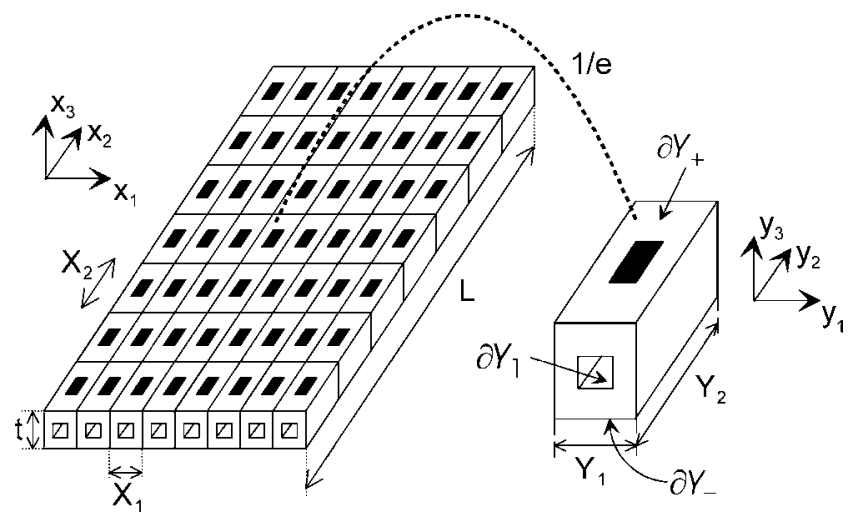

Fig. 2. Type of panel studied. make $\varepsilon \rightarrow 0$ first, and then $e \rightarrow 0$ using an appropriate sandwich plate theory. The interested reader is referred to the book of Lewinski and Telega [8], where the methods are widely presented, and to $[9,10]$. In [8], the different methods are applied to the bending of a symmetric stiffened panel, and in [9 11 ] to the membrane and bending behaviors of plates with honeycomb core and of corrugated plates with straight unidirectional stiffeners.

In practice, the choice of a homogenization method is dictated by the value of ratio $e / \varepsilon$. The panels studied here have values of $e$ and $\varepsilon$ that are on the same order of magnitude. From a classical study of a heterogeneous plate made of a large number of basic cells, one can identify the equivalent characteristics of the plate. Then, by comparing these characteristics and those given by different homogenization methods, it appears that the most accurate results are obtained when both small parameters simultaneously become vanishingly small [10]. This is in the same line as the conclusion drawn in [8]. The latter method will therefore be used here.

This method was initially presented in [7] and is exposed in detail in [8]. A similar approach was proposed in [12] to study the bending of symmetric plates with a variable thickness. In concrete terms, the homogenization method involves basic cell problems posed on the $3 \mathrm{D}$ period of the panel, i.e. including both the core and the facings. Therefore, as noted in [11], this method is more appropriate than the homogenization of the core into an equivalent medium separately (see [4] for cellular sandwich cores), even if the latter may incorporate core face sheet constraints [13].

Solving the basic cell problems provides the plate effective properties according to a Kirchhoff Love model. In the literature, approximated analytical solutions to the cell problems were presented in [8] for unidirectional periodic plates, and in [14 16] for various applications (stiffened plates, sandwich plates with honeycomb core and fiber reinforced plates).

The cell problems can also be solved numerically using a finite element model of the basic cell. One can find such results in [12] for the bending of symmetric stiffened plates, in [17] for unidirectional periodic plates and in [9 11] for plates with straight unidirectional stiffeners or honeycomb core. This method of resolution presents the advantage that it can be applied to any type of period and this approach was adopted here.

In the present work, the homogenization theory, based on the asymptotic expansion method, will briefly be described in Section 2. The basic cell problems and their numerical implementation will be presented shortly after. Then, in Section 3, the homogenization method will be applied to the different panels shown in Fig. 1. By comparing the response of the equivalent homogeneous plates with that given by detailed finite element models of sandwich panels, the accuracy of the method will be 
demonstrated in Section 4 for in-plane and pure bending types of loading. In Section 5, the effective properties of a fiber-reinforced plastic (FRP) honeycomb sandwich panel are computed and compared to those given in [18], in order to show the applicability of the homogenization method presented in this paper to such structures.

The main limitation of the homogenization method used here is that, at the leading order, it leads to an equivalent Kirchhoff Love homogeneous plate. For the structures studied in the present paper, it is likely that such a model will not be totally satisfactory. This problem will be dealt with in Section 6, where a simple method will be proposed to obtain the panel effective transverse shear stiffness, and finally get the properties of an equivalent Reissner Mindlin homogeneous plate.

The summation convention on repeated indices will be used throughout the paper. The Latin indices range from 1 to 3 , whereas the Greek indices range from 1 to 2 . In addition, dots and semi-colons will respectively note the scalar and double products of tensors, e.g.: $(\sigma \cdot n)_{i}=\sigma_{i j} n_{j}$ and $(a: e)_{i j}=a_{i j k l} e_{k l}$.

\section{The homogenization method}

\subsection{Main results}

Following $[7,8,10]$, the main steps of the homogenization method are exposed in this section.

The starting point is the formulation of the 3D problem of a thin structure with in-plane periodic components (the general case of a periodic structure in both directions of its plane is studied here). As mentioned in introduction, this problem involves two small parameters: $e$, which corresponds to the slenderness of the structure, and $\varepsilon$, associated to the rapid periodic variation of the material and geometrical properties in the in-plane directions. These two small parameters are of the same order of magnitude and are assumed to be equal. Thus, one can use the asymptotic expansion method with one small parameter for periodic plates.

Firstly, two scales are introduced: a microscopic one, which is the scale of the heterogeneities and of the thickness, and a macroscopic scale on which the size of the basic cell is very small. The corresponding variables are respectively: $y_{i}=x_{i} / e$ and $\left(z_{1}, z_{2}\right)=\left(x_{1}, x_{2}\right)$, where $(x)=\left(x_{1}, x_{2}, x_{3}\right)$ is the initial $3 \mathrm{D}$ variable. The operators of the 3D elasticity problem are then expressed as functions of these new variables. Next, the solution of this problem is searched under the form:

$$
\begin{aligned}
u(x)= & u^{0}\left(z_{1}, z_{2}\right)+e u^{1}\left(z_{1}, z_{2}, y_{1}, y_{2}, y_{3}\right) \\
& +e^{2} u^{2}\left(z_{1}, z_{2}, y_{1}, y_{2}, y_{3}\right)+\cdots
\end{aligned}
$$

where functions $u^{i}\left(z_{1}, z_{2}, y_{1}, y_{2}, y_{3}\right)$ are $Y_{1}$ and $Y_{2}$-periodic in variable $y, Y_{\alpha}=X_{\alpha} / e$ being a length of the period or basic cell $Y$ at the microscopic scale, see Fig. 2.

It will be considered that the first term $u^{0}\left(z_{1}, z_{2}\right)$ of the expansion in Eq. (1) has only a component in direction 3 , which amounts to assuming that the plate bending is prominent, the terms relative to the in-plane displacement being of the 1 th order in $e$, that is $u_{\alpha}^{0}=0$.

It then turns out that the $3 \mathrm{D}$ elasticity problem splits in a sequence of $2 \mathrm{D}$ microscopic problems, posed on the basic cell, and 2D macroscopic problems providing the overall plate response.

The main results of the method are now recalled, for more details, see $[7,8,10]$ :

- The solution of the leading order ( -1 th order) microscopic problem is (the solution is unique up to an additive constant $\hat{u}^{1}$, which corresponds to a rigid body translation):

$u_{i}^{1}=-\hat{y}_{3} \frac{\partial u_{3}^{0}}{\partial z_{\alpha}} \delta_{i \alpha}+\hat{u}_{i}^{1}\left(z_{1}, z_{2}\right)$

where $\delta_{i \alpha}$ is the Kronecker symbol, and where $\hat{y}_{3}=$ $y_{3}-y_{3}$ with $y_{3}=\left(1 /\left|Y^{*}\right|\right) \int_{Y^{*}} y_{3} \mathrm{~d} y_{1} \mathrm{~d} y_{2} \mathrm{~d} y_{3}$, and $\left|Y^{*}\right|$ represents the volume of the solid part $Y^{*}$ of $Y$. Then, $\hat{y}_{3}$ is the distance in direction 3 from the mid-plane of the panel, such that $y_{3}=y_{3}$ or $\hat{y}_{3}=0$. In the following, the overall plate behavior will be formulated with respect to the mid-plane.

- The leading order macroscopic problem generalizes the Kirchhoff Love theory, the transverse and inplane displacements being $u_{0}^{3}$ and $\hat{u}_{\alpha}^{1}$. The corresponding homogenized (or effective) constitutive relations are obtained from the solution of the 0th order microscopic problem, which will be exposed in Section 2.2 .

\subsection{The basic cell problems}

Let us introduce membrane macrodeformations $E$ and curvature macrodeformations $K$ defined as:

$\left\{\begin{array}{l}E_{\alpha \beta}=\varepsilon_{z_{\alpha \beta}}\left(\hat{u}^{1}\right) \\ K_{\alpha \beta}=-\frac{\partial^{2} u_{0}^{3}}{\partial z_{\alpha} \partial_{\beta}}\end{array}\right.$

where $\varepsilon_{z}$ denotes the strain operator corresponding to the macroscopic variable $z$.

The 0 th order microscopic problems are posed on the basic cell (see Fig. 2). The upper and lower facings are respectively noted $\partial Y_{+}$and $\partial Y_{-}$, while $\partial Y_{1}$ stands for the internal surfaces, which are not loaded. Denoting $a$ the elastic moduli tensor, $\operatorname{div}_{y}$ and $\varepsilon_{y}$ the divergence and strain operators with respect to the microscopic variable $y$, the basic cell problems consist in finding the fields $u^{\text {per }}$, $\varepsilon, \sigma^{1}$ such that: 


$$
\left\{\begin{array}{l}
\operatorname{div}_{y} \sigma^{1}=0 \\
\sigma^{1}=a(y): \varepsilon \\
\varepsilon_{\alpha \beta}=E_{\alpha \beta}+\hat{y}_{3} K_{\alpha \beta}+\varepsilon_{y_{\alpha \beta}}\left(u^{\text {per }}\right) \\
\varepsilon_{i 3}=\varepsilon_{y_{i 3}}\left(u^{\text {per }}\right) \\
\sigma^{1} \cdot n=0 \text { on } \partial Y_{ \pm} \cup \partial Y_{1} \\
u^{\text {per }} y_{1} y_{2} \text { per and } \sigma^{1} \cdot n \text { anti-per }
\end{array}\right.
$$

where 'per' means $Y_{1}$ and $Y_{2}$-periodic in variable $y$, and anti-per means that $\sigma^{1} \cdot n$ are opposite on opposite sides of $\partial Y$.

The data of problem (4) are the macrodeformations $E$ and $K$, and due to linearity, its solution is, up to a constant for the displacement:

$$
\left\{\begin{array}{l}
u_{i}^{2}=\chi_{i}^{E_{\alpha \beta}}(y) E_{\alpha \beta}+\chi_{i}^{K_{\alpha \beta}}(y) K_{\alpha \beta} \\
\sigma_{i j}^{1}=F_{i j \alpha \beta} E_{\alpha \beta}+G_{i j \alpha \beta} K_{\alpha \beta} \\
F_{i j \alpha \beta}=a_{i j k l} \frac{\partial x_{\alpha \beta}}{\partial y_{l}}+a_{i j \alpha \beta} \\
G_{i j \alpha \beta}=a_{i j k l} \frac{\partial x_{k}}{\partial y_{l}}+\hat{y}_{3} a_{i j \alpha \beta}
\end{array}\right.
$$

The overall plate behavior is defined from the macroscopic in-plane stress resultants and stress couples associated with $\sigma^{1}$. The latter are obtained through integration over the thickness and calculated using the following averaging method in period mid-plane $\hat{y}_{3}=0$ :

$$
\left\{\begin{array}{l}
N_{\alpha \beta}^{1}=\left\langle\sigma_{\alpha \beta}^{1}\right\rangle ; \quad M_{\alpha \beta}^{1}=\left\langle\hat{y}_{3} \sigma_{\alpha \beta}^{1}\right\rangle \\
\langle\cdot\rangle=\frac{1}{|Y|} \int_{Y^{*}} \cdot \mathrm{d} y_{1} \mathrm{~d} y_{2} \mathrm{~d} y_{3} \quad \text { with }|Y|=Y_{1} Y_{2}
\end{array}\right.
$$

The homogenized constitutive equation can then be put in the form:

$$
\left\{\begin{array}{l}
\left\{N^{1}\right\} \\
\left\{M^{1}\right\}
\end{array}\right\}=\left[\begin{array}{cc}
{[A]} & {[B]} \\
{[B]^{t}} & {[D]}
\end{array}\right]\left\{\begin{array}{l}
\{E\} \\
\{K\}
\end{array}\right\}
$$

with the matrices $[A],[B],[D]$ easily obtained from the tensors $F$ and $G$ introduced in Eq. (5), and the expressions given in Eq. (6).

Obtaining functions $\chi^{E_{\alpha \beta}}$ and $\chi^{K_{\alpha \beta}}$ requires to solve 6 cell problems similar to problem (4), where one successively considers that the only non-zero data is a component of the membrane macrodeformation $E$ or curvature $K$.

\subsection{Numerical solving of homogenization problems}

The basic cell problems (4) have the same characteristics as those dealt with for the homogenization of the elastic behavior of periodic composite materials (macroscopic deformation, periodicity conditions), and for which different finite element solution approaches are available in the literature [19 23].

One can mainly distinguish 2 solution techniques for the cell problems, both of them using the finite element method. The first technique consists in taking macrodeformations as problem input data and in working with stress field $\tau^{1}=a(y): \varepsilon_{y}\left(u^{\mathrm{per}}\right)$. The macrodeformations amount to body forces and surface loads on the edges, and the calculation of the global force vector is therefore rather tedious.

As a result, we chose a second technique, where one considers a stress field $\sigma^{1}$, and whose starting point is the following variational formulation of problem (4):

$$
\left\{\begin{array}{c}
\text { Find } u^{\text {per }} y_{1} y_{2} \text { per and } E, K \text { such that } \forall \tilde{u} y_{1} y_{2} \text { per } \\
\text { and } \widetilde{E}, \widetilde{K} \\
\int_{Y^{*}}\left(\widetilde{E}+y_{3} \widetilde{K}+e_{y}\left(\tilde{u}^{\text {per }}\right)\right): a:\left(E+y_{3} K+e_{y}\left(u^{\text {per }}\right)\right) \\
\mathrm{d} y=|Y|\left(\widetilde{E}: N^{1}+\widetilde{K}: M^{1}\right)
\end{array}\right.
$$

This technique was initially described in [20] for composite materials, and was used in $[9,10]$ for plate problems. The discretized field at the finite element level is $u^{\text {per }}$, but macroscopic deformations $E$ and $K$ are considered as additional degrees of freedom. These macroscopic degrees of freedom are associated with a virtual node connected to all the elements of the mesh. The matrix relation between the deformations and the degrees of freedom of the elements is then modified to satisfy Eq. (4) ${ }_{3}$ and (4) ${ }_{4}$ as shown below (let $\left\{u_{e}^{\text {per }}\right\}$ denote the degrees of freedom associated with $u^{\text {per }}$ for the element considered):

$$
\left\{\begin{array}{c}
\varepsilon_{11} \\
\varepsilon_{22} \\
\varepsilon_{33} \\
2 \varepsilon_{12} \\
2 \varepsilon_{23} \\
2 \varepsilon_{13}
\end{array}\right\}=\left[[B]\left[\begin{array}{cccccc}
1 & 0 & 0 & \hat{y}_{3} & 0 & 0 \\
0 & 1 & 0 & 0 & \hat{y}_{3} & 0 \\
0 & 0 & 0 & 0 & 0 & 0 \\
0 & 0 & 1 & 0 & 0 & \hat{y}_{3} \\
0 & 0 & 0 & 0 & 0 & 0 \\
0 & 0 & 0 & 0 & 0 & 0
\end{array}\right]\right]\left\{\begin{array}{c}
\left\{u_{e}^{\mathrm{per}}\right\} \\
E E\} \\
\{K\}
\end{array}\right\}
$$

where $[B]$ is the element usual matrix, and $\{E\}^{t}=\left\{E_{11}\right.$, $\left.E_{22}, 2 E_{12}\right\},\{K\}^{t}=\left\{K_{11}, K_{22}, 2 K_{12}\right\}$.

At this stage, let us recall that the origin of $\hat{y}_{3}$ corresponds to the reference plane with respect to which the homogenized characteristics are obtained.

On the other hand, the periodicity of $u^{\mathrm{per}}$ is taken into account by eliminating the redundant degrees of freedom, see [23] for example.

Finally, Eq. (9) yields the following set of matrix equations, where $\left\{N^{1}\right\}^{t}=\left\{N_{11}, N_{22}, N_{12}\right\}$, and $\left\{M^{1}\right\}^{t}=$ $\left\{M_{11}, M_{22}, M_{12}\right\}$ :

$[S]\left\{\begin{array}{c}\left\{u^{\text {per }}\right\} \\ \{E\} \\ \{K\}\end{array}\right\}=|Y|\left\{\begin{array}{c}\{0\} \\ \left\{N^{1}\right\} \\ \left\{M^{1}\right\}\end{array}\right\}$

where $[S]$ is the stiffness matrix of the structure calculated from Eq. (9) and from the local constitutive equation.

As a consequence, it appears that the dual variables to macroscopic deformation $\{E\}$ and $\{K\}$ are respectively $|Y|\left\{N^{1}\right\}$ and $|Y|\left\{M^{1}\right\}$, wherefrom a simple way to obtain the homogenized constitutive equation. Indeed, noting this equation in the form given in Eq. (7) and by successively imposing a component of $\left\{\{E\}^{t},\{K\}^{t}\right\}$ in 
Eq. (10) to be equal to unity and the others to be zero, displacements $\chi^{E_{\alpha \beta}}$ and $\chi^{K_{\alpha \beta}}$ are the solution $u^{\text {per }}$. The calculation of the reaction forces associated with the boundary conditions imposed on the macroscopic degrees of freedom makes it possible to build the stiffness matrix in Eq. (7) column by column.

All these different steps were implemented in a finite element program. In practice, quadratic $3 \mathrm{D}$ solid finite elements are taken to mesh the cell in order to model the bending phenomena properly. In addition, the uniqueness of the solution to Problem (4) is ensured by zeroing $u^{\text {per }}$ at one node of the cell.

Because of the one-directional periodicity of the stiffeners, the homogenization problems are bidimensional, in plane $\left(x_{1}, x_{3}\right)$. However, the finite element program was designed for more general applications (periodic panels in two directions, see Fig. 2) and the modifications mentioned above on the element matrices have only been made on the $3 \mathrm{D}$ solid elements. The model used was therefore fully $3 \mathrm{D}$, with only one element in the direction $x_{2}$. In the following, the finite element computation of the plate effective properties will be called the numerical homogenization method.

We will also use an analytical homogenization method. Since the cell problems are bidimensional, kinematical assumptions over the thickness make it possible to reduce the homogenization problem to that of a one-dimensional beam. Then, analytical expressions for the homogenized characteristics of the plate can be obtained [8]. Let us remark that the range of applications for such a method is narrow, because analytical expressions cannot be obtained for any shape of stiffeners. In particular, this method will only be used here for straight stiffeners, the other structures being studied by numerical method. The analytical expressions of the homogenized characteristics are given in Appendix A.

We are now going to apply the numerical and analytical homogenization methods described above to the different panels shown in Fig. 1.

\section{Membrane and bending behaviors of the different structures}

It is recalled that the objective of the present study is to calculate the static mechanical behavior of each structure in order to compare the stiffnesses of the alveolar geometries with that of the stiffened panel, for a given mass.

The usual panel taken as a reference for this study is a portion of stiffened panel between the main girders of the steel hull of a passengers ship. This stiffened plate is made out of 5-mm thick steel longitudinally reinforced by bulb flats HP $100 \times 6$ (see Fig. 3).

The mechanical properties for steel are $E=210 \mathrm{GPa}$, $v=0.3$.
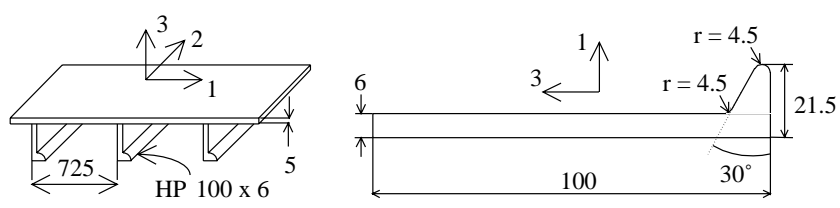

Fig. 3. Reference stiffened panel and stiffener dimensions, in $\mathrm{mm}$.

\subsection{Comparison indicators}

In order to avoid the tedious comparison of all the components of the overall behavior matrices obtained for the different panels, it is useful to consider some characteristic values taken as indicators.

First, let us mention that the panel equivalent characteristics are determined with respect to the reference plane defined by $y_{3}=y_{3}$ for all the panels except the stiffened one, for which the reference plane is the midplane of the carrier plate. On the other hand, following $[7,8]$ and due to the symmetry properties of the cells, it can be shown that, for all the panels, the terms $\alpha \beta \gamma \delta$ representing respectively the membrane, membrane bending coupling and bending effective stiffnesses in matrices $[A],[B],[D]$ are zero if index 1 or 2 appears an odd number of times, and matrix $[B]$ is zero except for the stiffened plate.

Considering the compliance matrices $[A]^{-1}$ and $[D]^{-1}$, one can classically [24] define moduli $E_{\alpha}^{m, f}$ and $G_{12}^{m, f}$ respectively associated with in-plane and bending behaviors (membrane and bending moduli):

$$
\begin{gathered}
{[A]^{-1}=\frac{1}{t_{\text {ref }}}\left[\begin{array}{ccc}
1 / E_{1}^{m} & -v_{21}^{m} / E_{2}^{m} & 0 \\
-v_{12}^{m} / E_{1}^{m} & 1 / E_{2}^{m} & 0 \\
0 & 0 & 1 / G_{12}^{m}
\end{array}\right]} \\
{[D]^{-1}=\frac{12}{t_{\text {ref }}^{3}}\left[\begin{array}{ccc}
1 / E_{1}^{f} & -v_{21}^{f} / E_{2}^{f} & 0 \\
-v_{12}^{f} / E_{1}^{f} & 1 / E_{2}^{f} & 0 \\
0 & 0 & 1 / G_{12}^{f}
\end{array}\right]}
\end{gathered}
$$

Direction 1 is taken perpendicular to the corrugation (see Fig. 1), and thickness $t_{\text {ref }}$ equal to $5 \mathrm{~mm}$, like the thickness of the sheet used for the reference panel.

The different panels can then be sorted by membrane moduli $\left(E_{1}^{m}, E_{2}^{m}, G_{12}^{m}\right)$ and by bending moduli $\left(E_{1}^{f}, E_{2}^{f}\right.$, $\left.G_{12}^{f}\right)$. These quantities are directly comparable to steel Young's and shear moduli, and can readily be interpreted by engineers.

\subsection{Implementation of the homogenization method}

\subsubsection{Introduction}

The geometry of the reference panel is known (see Fig. 3), but that of the alveolar panels is to be determined. Since our objective is to compare the ratios between the equivalent stiffnesses and the mass of the different structures, our methodology will be as follows. 
After calculating the stiffness moduli $E_{\alpha}^{m, f}$ and $G_{12}^{m, f}$ of the reference stiffened panel using the numerical homogenization method, the panel with straight stiffeners will be considered. Indeed, the analytical homogenization method can be implemented on this panel, making it possible to carry out a parametric study of its different geometric characteristics. Geometry for the panel with straight stiffeners will be determined such that its stiffness moduli lie within 5\% of those of the reference panel. The other panel geometries will then be set in such a way that the mass per unit area of the panels be close to that of the panel with straight stiffeners. Finally, the numerical homogenization method will be applied to all the alveolar panels.

\subsubsection{Study of the panel with straight stiffeners}

The purpose here is to determine the geometry of the panel with straight stiffeners according to the procedure explained above. To this aim, a parametric pre-study is made using an analytical method of homogenization (the expressions used are given in Appendix A). The parameters of this study are total thickness $t$ of the panel, thickness $t_{\mathrm{f}}$ of the facings, thickness $t_{\mathrm{c}}$ of the core and distance $p$ between two stiffeners (see Fig. 4). By varying these geometric parameters in turn, the others being fixed, their respective influence on the panel overall membrane and bending behaviors can be assessed. The values that parameters $t, t_{\mathrm{f}}, t_{\mathrm{c}}$ and $p$ should be given can then readily be determined, for stiffness moduli $E_{\alpha}^{m, f}$ and $G_{12}^{m, f}$ to be at least within 5\% of those of the stiffened panel.

After computation, it appears that bending modulus $E_{2}^{f}$ is mainly driven by panel total thickness $t$, and that the three other parameters, $t_{\mathrm{f}}, t_{\mathrm{c}}$ and $p$ essentially affect the membrane moduli. A $60 \mathrm{~mm}$ thickness $t$ is necessary to meet the bending stiffness $E_{2}^{f}$ of the stiffened plate. As for the other parameters, $t_{\mathrm{f}}, t_{\mathrm{c}}$ and $p$, they must respectively be set to $2.4 \mathrm{~mm}, 1 \mathrm{~mm}$ and $45 \mathrm{~mm}$ to obtain the membrane moduli $E_{1}^{m}$ and $E_{2}^{m}$ of the stiffened panel. One finally gets a cell period whose geometric characteristics are defined in Fig. 4.

\subsubsection{Geometric definition of the three other types of} alveolar panels (hat type, triangular, curvilinear)

Geometry of the other alveolar panels was set in order for the panels' mass per unit area to be close to that

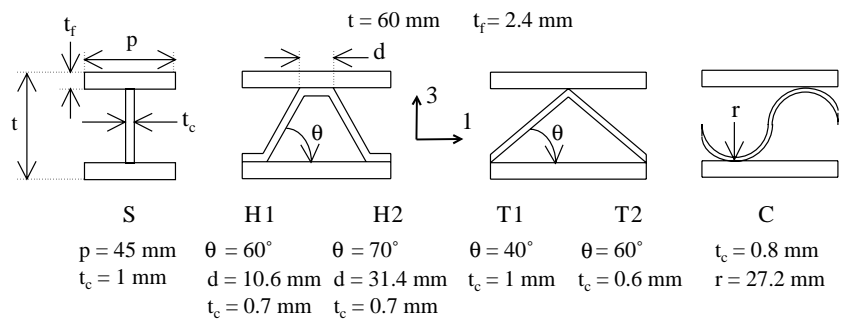

Fig. 4. Alveolar geometries studied. of the panel with straight stiffeners. Moreover, panel thickness $t$ and facings thickness $t_{\mathrm{f}}$ were taken identical for all the structures, so as to highlight the role of the core only. The cell periods defined are shown in Fig. 4.

\subsubsection{Numerical homogenization of the alveolar panels}

The geometric characteristics of the cell periods studied are presented Fig. 4.

These cell periods are studied using the numerical homogenization method. An example of the deformed

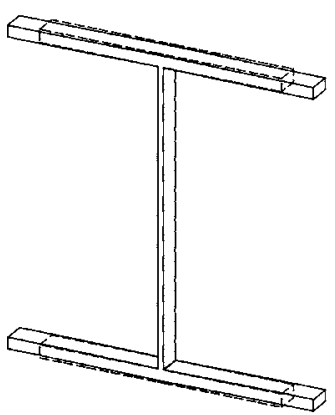

(a)

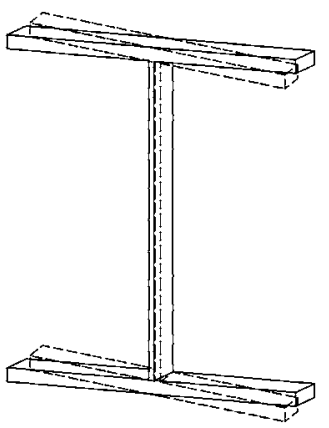

(c)

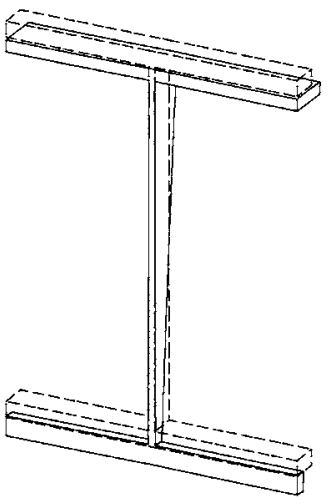

(e)

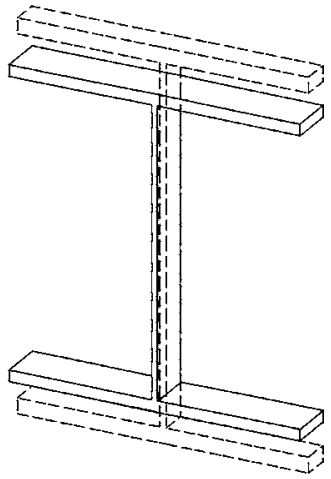

(b)

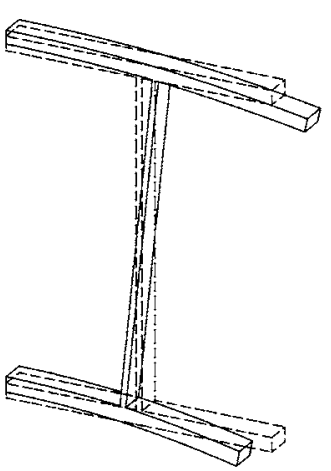

(d)

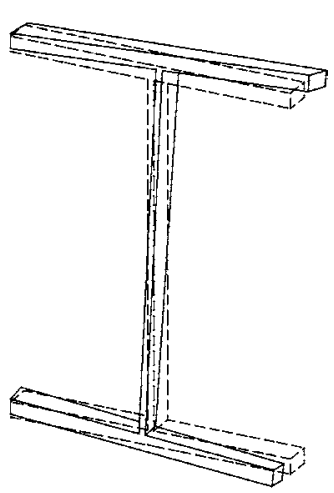

(f)
Fig. 5. Deformed shapes obtained from solving the basic cell problems for the panel with straight stiffeners: initial configuration shown in broken line, deformed shape in solid line. (a) $\chi^{E_{11}}$, (b) $\chi^{E_{22}}$, (c) $\chi^{E_{12}}$, (d) $\chi^{K_{11}}$, (e) $\chi^{K_{22}}$ and (f) $\chi^{K_{12}}$. 
Table 1

Panel membrane and bending homogenized characteristics

\begin{tabular}{|c|c|c|c|c|c|c|c|c|c|}
\hline \multirow{3}{*}{$\begin{array}{l}\text { Structure (type } \\
\text { of stiffener) }\end{array}$} & \multicolumn{4}{|c|}{ Membrane behavior } & \multicolumn{4}{|c|}{ Bending behavior } & \multirow{3}{*}{$\begin{array}{l}\text { Mass per unit } \\
\text { area }\left(\mathrm{kg} / \mathrm{m}^{2}\right)\end{array}$} \\
\hline & \multicolumn{3}{|c|}{ Moduli (GPa) } & \multirow{2}{*}{ Poisson's ratio, $v_{12}^{m}$} & \multicolumn{3}{|c|}{ Moduli (10E3 GPa) } & \multirow{2}{*}{ Poisson's ratio, $v_{12}^{f}$} & \\
\hline & $E_{1}^{m}$ & $E_{2}^{m}$ & $G_{12}^{m}$ & & $E_{1}^{f}$ & $E_{2}^{f}$ & $G_{12}^{f}$ & & \\
\hline Ref & 213.5 & 254.9 & 80.91 & 0.0046 & 13.43 & 90.48 & 4.808 & 0.0008 & 47.64 \\
\hline $\mathrm{S}$ & 205.5 & 253.1 & 77.68 & 0.2436 & 80.90 & 86.59 & 30.94 & 0.2803 & 47.31 \\
\hline $\mathrm{H} 1$ & 208.1 & 252.8 & 84.54 & 0.2469 & 81.91 & 88.20 & 31.68 & 0.2786 & 47.25 \\
\hline $\mathrm{H} 2$ & 213.1 & 252.7 & 85.54 & 0.2530 & 83.83 & 90.69 & 32.92 & 0.2773 & 47.23 \\
\hline $\mathrm{T} 1$ & 205.7 & 256.1 & 89.94 & 0.2409 & 80.90 & 86.88 & 30.93 & 0.2794 & 47.87 \\
\hline $\mathrm{T} 2$ & 205.3 & 251.7 & 82.47 & 0.2447 & 80.84 & 86.35 & 30.91 & 0.2808 & 47.05 \\
\hline $\mathrm{C}$ & 205.4 & 254.4 & 85.76 & 0.2423 & 81.07 & 89.68 & 30.89 & 0.2712 & 47.54 \\
\hline
\end{tabular}

Moduli $E_{\alpha}^{m, f}$ and $G_{12}^{m, f}$ for an equivalent $5 \mathrm{~mm}$ thick plate.

shapes corresponding to the solution of the basic cell problems is shown in Fig. 5.

The results concerning the panels' stiffness moduli and Poisson's ratios in membrane and bending behaviors are gathered in Table 1.

\subsection{Results analysis}

First of all, it is to be noticed that the discrepancies in the mass per unit area reach $1.2 \%$ at most. Then, comparing the different structures from the point of view of their ratios of effective stiffness over mass comes down to comparing their equivalent stiffnesses.

As opposed to the reference stiffened panel, the alveolar panels do not exhibit significant differences in their membrane stiffnesses $E_{1}^{m}, E_{2}^{m}, G_{12}^{m}$ nor in their longitudinal bending stiffnesses $E_{2}^{f}$. However, their bending moduli $E_{1}^{f}$ are much higher (in the orthogonal plane to corrugation) and so are the torsion moduli $G_{12}^{f}$. In fact, the pure bending behavior of alveolar panels is quasiisotropic, which means that $E_{1}^{f}$ and $E_{2}^{f}$ are on the same order of magnitude and that $G_{12}^{f}$ is within a $\left(E^{f} / 2(1+\right.$ $v)$ ) ratio, where $v=0.3$. On the contrary, the traditional stiffened plate features very different $E_{1}^{f}$ and $E_{2}^{f}$ moduli: the effect of the stiffeners is most significant in the corrugation direction (direction 2, see Fig. 1). The stiffened panel therefore exhibits a strongly anisotropic bending behavior.

On the other hand, the comparison of the six alveolar geometries between them shows that they have almost identical effective stiffnesses. This result reveals the prominent role played by the facings on the overall membrane and bending behaviors of an alveolar panel: as mentioned above about the straight stiffeners, the facings thickness drives the panel membrane behavior and the facings spacing drives the bending behavior. In comparison, the core shape has practically no influence on the membrane or bending stiffnesses.

Another way to pinpoint the important role played by the facings on the panel overall behavior consists in using the classical laminated plate theory to assess the equivalent behavior of a multilayered composite plate made of two facings and one intermediate layer whose Young's modulus is negligible compared to that of the facings. One obtains:

$$
\begin{aligned}
& E_{1}^{m}=E_{2}^{m}=\frac{2 t_{\mathrm{f}}}{h_{\mathrm{ref}}} E=201.6 \mathrm{GPa} \\
& G_{12}^{m}=\frac{2 t_{\mathrm{f}}}{h_{\mathrm{ref}}} G=77.54 \mathrm{GPa} \\
& E_{1}^{f}=E_{2}^{f}=\frac{h_{\mathrm{ref}}^{3}-\left(h_{\mathrm{ref}}-2 t_{\mathrm{f}}\right)^{3}}{h_{\mathrm{ref}}^{3}} E=80.31 \times 10 \mathrm{E} 3 \mathrm{GPa} \\
& G_{12}^{f}=\frac{h_{\mathrm{ref}}^{3}-\left(h_{\mathrm{ref}}-2 t_{\mathrm{f}}\right)^{3}}{h_{\mathrm{ref}}^{3}} G=30.89 \times 10 \mathrm{E} 3 \mathrm{GPa}
\end{aligned}
$$

Then, it appears that the core has only a significant contribution in the case of loads in the corrugation direction and for in-plane shear of the panel.

\section{Validation of the homogenization method for membrane and pure bending loadings}

\subsection{Presentation of the method}

In order to validate the homogenization method presented in Section 3, loading cases corresponding to simple macroscopic resultant stresses will be considered. At first, the response of the real heterogeneous panel predicted by the finite element method will be compared to the analytical solution of the same problem posed on the equivalent homogeneous plate. This procedure will make it possible to identify the equivalent characteristics of the homogeneous panel. These values will then be taken as references and will be checked against those obtained from the numerical homogenization method.

With the above objective in mind, let us consider a panel of finite dimension in one direction and of infinite dimension in the perpendicular direction, so that the problem is simplified into the one-dimensional cylindrical bending of an equivalent plate. 
As far as the finite element model of the real panel is concerned, the cylindrical bending conditions are imposed in the following way:

- for the study in plane 13, only one finite element is taken in direction 2 since the structure is invariant in this direction, and the translation and rotation degrees of freedom of two nodes A and B facing each other in direction 2 are made equal. See Fig. 6(a) for the panel with $60^{\circ}$ (T2) triangular stiffeners. All the following finite element analyses were run with the commercial package Samcef [25]. The meshes were made of 4-node Mindlin elements, and a perfect bonding between the facings and the core was assumed.

- for the study in plane 23 , one considers a one-period length in direction 1 because the structure is not invariant in this direction. The cylindrical bending conditions are written at nodes A and B located on the edges of the cell period. See Fig. 6(b).

\subsection{Membrane loading}

The loading and boundary conditions on the heterogeneous panel in plane 13 are described in Fig. 7(a).

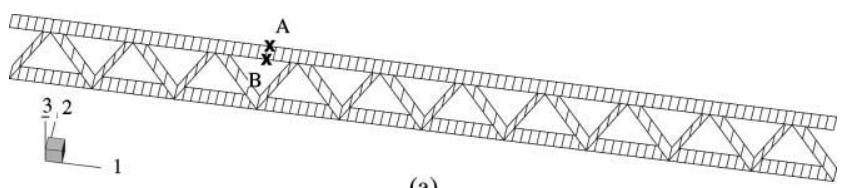

(a)

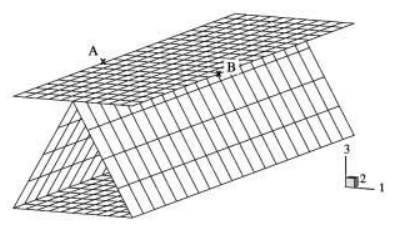

(b)

Fig. 6. Finite element model used for the study (a) in plane 13 and (b) in plane 23 .

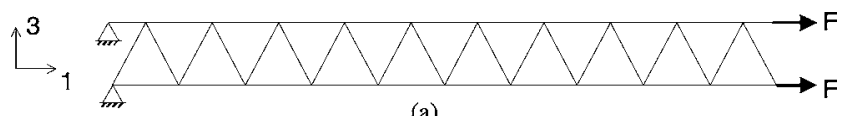

(a)

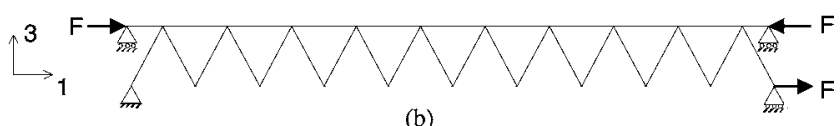

(b)

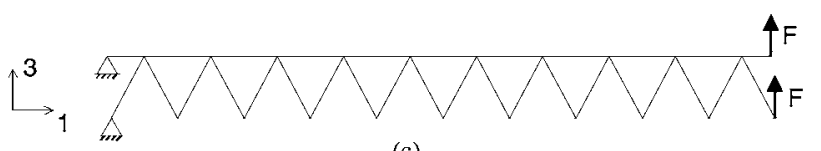

(c)

Fig. 7. Loading and boundary conditions in (a) membrane, (b) pure bending and (c) simple bending.
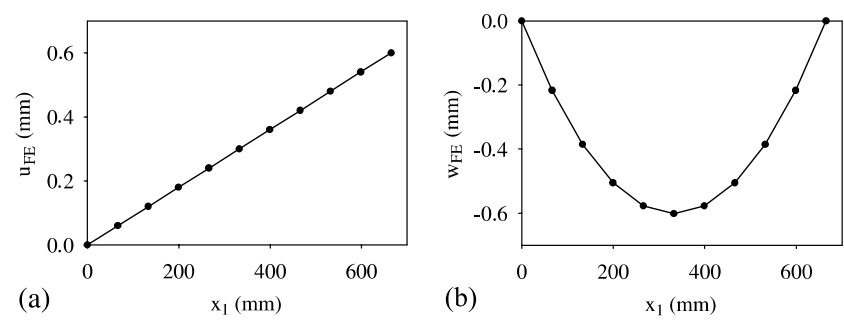

Fig. 8. Panel response with T2 stiffeners in (a) membrane, (b) pure bending loadings.

The analysis is made on a 10-period model, and force $F$ is calculated such that $N_{11}=10 \mathrm{E} 3 \mathrm{~N} / \mathrm{mm}$.

The response of the structure is then estimated using the finite element method, and half-sum $u_{\mathrm{FE}}$ of the axial displacements of the two facings at a given position $x_{1}$ is computed for comparison with the analytical solution of the homogeneous equivalent plate problem.

The membrane and bending behaviors of the equivalent homogeneous plate being uncoupled, and matrix $[A]$ in Eq. (7) being such that $A_{1112}=A_{2212}=0$, the analytical solution is given as:

$u=\frac{N_{11}}{A_{1111}} x_{1}$

As an example, the results obtained for the panel with $\mathrm{T} 2$ triangular stiffeners are presented. If $u_{\mathrm{FE}}$ is plotted as a function of $x_{1}$, one gets the curve shown in Fig. 8(a), and this curve can very well be approximated as a straight line. In addition, the slope of this straight line can be identified using the least-square method, which yields $A_{1111}=1.108 \times 10 \mathrm{E} 6 \mathrm{~N} / \mathrm{mm}$.

On the other hand, according to the results about the T2 panel (see Table 1), the value obtained from homogenization is:

$A_{1111}=h_{\text {ref }} \frac{E_{1}^{m}}{1-\left(v_{12}^{m}\right)^{2} \frac{E_{2}^{m}}{E_{1}^{m}}}=1.108 \times 10 \mathrm{E} 6 \mathrm{~N} / \mathrm{mm}$

There is obviously an excellent agreement between the value of the effective stiffness determined by the finite element analysis of the real heterogeneous panel and that calculated by the homogenization method.

\subsection{Pure bending loading}

In plane 13, the boundary conditions and forces shown in Fig. 7(b) impose a pure bending loading. Force $F$ is such that $M_{11}=-1000 \mathrm{~N}$.

The results given by the finite element method are analyzed after determining $w_{\mathrm{FE}}$ defined as the half-sum of the deflections of the two facings at a given position $x_{1}$.

For the homogeneous plate, matrix $[D]$ in Eq. (7) being such that $D_{1112}=D_{2212}=0$, the analytical solution 
is given as (where $\ell$ represents the length of the panel studied):

$w=\frac{M_{11}}{2 D_{1111}} x_{1}\left(\ell-x_{1}\right)$

For a 10-period-long panel with T2 stiffeners, the results for $w_{\mathrm{FE}}$ are shown in Fig. 8(b). By determining coefficient $D_{1111}$ such that Eq. (15) best represents $w_{\mathrm{FE}}$ in the least-square sense, one gets $D_{1111}=9.195 \times 10 \mathrm{E} 8$ $\mathrm{N} \cdot \mathrm{mm}$. And the value obtained from homogenization is (see Table 1):

$D_{1111}=\frac{\left(h_{\mathrm{ref}}\right)^{3}}{12} \frac{E_{1}^{f}}{1-\left(v_{12}^{f}\right)^{2} \frac{E_{2}^{f}}{E_{1}^{f}}}=9.195 \times 10 \mathrm{E} 8 \mathrm{~N} \cdot \mathrm{mm}$

Like for membrane loading, there is an excellent agreement between the bending effective stiffness calculated on the real panel and that obtained from the homogenization method.

These results are as satisfactory as those shown above for the other membrane and bending characteristics, and this holds for all the panel types studied here. Another example of the accuracy of the homogenization method used in the present paper can be found in $[9,10]$ where the example of a honeycomb plate made of steel is treated. In order to show the applicability of the homogenization method to composite structures, an example with FRP materials is treated in Section 5.

\section{Homogenization of a fiber-reinforced plastic honey- comb sandwich panel}

As an example of FRP structure, we consider the case of a honeycomb sandwich panel for highway decks, which was studied in [18]. This structural panel consists of a sinusoidal wave core configuration in the plane, extending vertically between face laminates. Thus, the panel exhibits in-plane periodic structure, and Fig. 9(a) presents its basic cell, the upper facing being not represented.

The geometric data of the basic cell are:

- thicknesses, total: $t=126.52 \mathrm{~mm}$, face laminates: $t_{\mathrm{f}}=$ $10.87 \mathrm{~mm}$, core: $t_{\mathrm{c}}=2.28 \mathrm{~mm}$,

- in plane dimensions: $p_{1}=101.6 \mathrm{~mm}, p_{2}=110.72$ $\mathrm{mm}$.

The core has a sinusoidal wave configuration, with an amplitude $2 h$ (with $h=25.4 \mathrm{~mm}$ ) and $p_{2}=4 h+4 t_{\mathrm{c}}$, see Fig. 9(a), one half of the core wall being modeled on the basic cell boundaries normal to direction 2 .

The core and face laminates are constituted with Eglass fibers and polyester resin. The core is made with a single layer of continuous strand mat with continuous randomly oriented fibers, while the face laminates include four types of fiber layers, see [18]. However, to solve the basic cell problems, it is indeed sufficient to consider the core (with its real sinusoidal geometry) and laminates as homogeneous layers. So we use here the equivalent properties given in [18], and obtained from a micromechanics approach for each layer, combined with classical lamination theory for the facings. These material properties are listed in Tables 2 and 3, and are taken from Tables 3 and 4 of [18] (in Table 2, the direction 3 is normal to the core layer, while in Table 3, the directions 1 and 2 correspond to those of Fig. 9(a)).

Let us mention that given that the basic cell problems are $3 \mathrm{D}$, a $3 \mathrm{D}$ constitutive behavior has to be defined from the data of Tables 2 and 3. So the following assumptions were made for the core properties $\left(E_{3}=E_{1}=E_{2}\right.$,

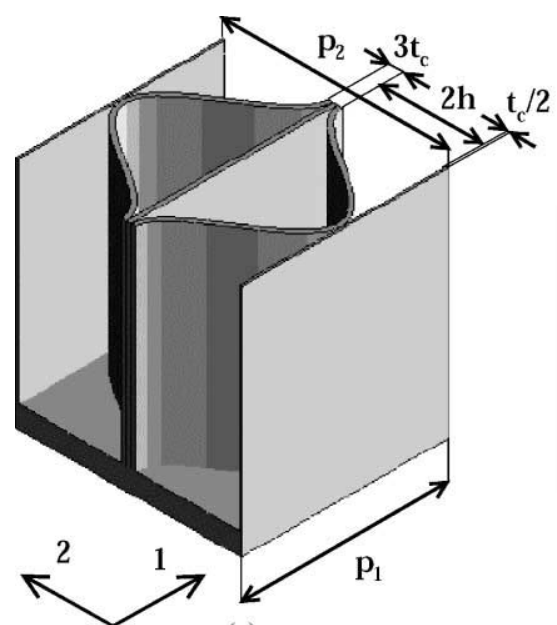

(a)

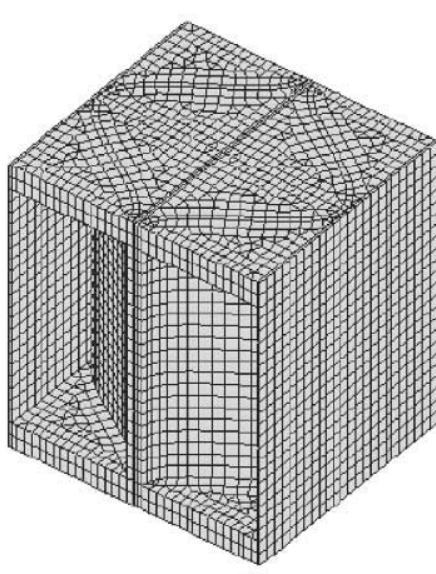

(b)

Fig. 9. Basic cell geometry of the FRP honeycomb sandwich panel (a) and its finite element modeling (b). 
Table 2

Engineering constants for the core material

\begin{tabular}{llllll}
\hline$E_{1}(\mathrm{GPa})$ & $E_{2}(\mathrm{GPa})$ & $G_{12}(\mathrm{GPa})$ & $G_{23}(\mathrm{GPa})$ & $v_{12}$ & $v_{23}$ \\
\hline 11.79 & 11.79 & 4.21 & 2.97 & 0.402 & 0.388 \\
\hline
\end{tabular}

Table 3

Engineering constants for face laminates

\begin{tabular}{llll}
\hline$E_{1}(\mathrm{GPa})$ & $E_{2}(\mathrm{GPa})$ & $G_{12}(\mathrm{GPa})$ & $v_{12}$ \\
\hline 19.62 & 12.76 & 3.76 & 0.302 \\
\hline
\end{tabular}

$G_{13}=G_{23}$ and $v_{13}=v_{23}$ ), while for the face laminates we took $E_{3}=E_{2}, v_{13}=v_{23}=v_{12}$ and $G_{12}=G_{23}=G_{13}$. One can easily verify that these assumptions have a negligible effect on effective properties since it's mainly the in-plane behavior of the core and the facings which is involved in the loadings of the basic cell problems. The mesh used for the numerical homogenization method is shown in Fig. 9(b).

From (11), with $t_{\text {ref }}=126.52 \mathrm{~mm}$ i.e. the panel total thickness, the effective moduli of the sandwich structure are computed. Table 4 shows the results obtained with the numerical 3D homogenization method, in comparison to those of [18]. In [18], in a first step, an analytical solution is used for the evaluation of equivalent core properties, and then classical lamination theory provides the sandwich panel effective properties, considering the structure as a three layers laminated system. Therefore, skin core interactions are neglected, which yields underestimate of stiffness, as noted in [11]. These effects are rigorously taken into account in the method used in this paper, through the 3D modeling used in the basic cell problems. From Table 4, it may be seen that there is not a significant difference between our approach and the results of [18]. However, our approach is more accurate because it enables to compute local distribution of stresses. Thus, once the computations are made on the equivalent homogeneous plate, these local stresses are simply obtained by a linear combination of the obtained macroscopic strains and the solution of the basic cell problems (see e.g. Fig. 10 the von-Mises stress distribution for a given unit curvature).
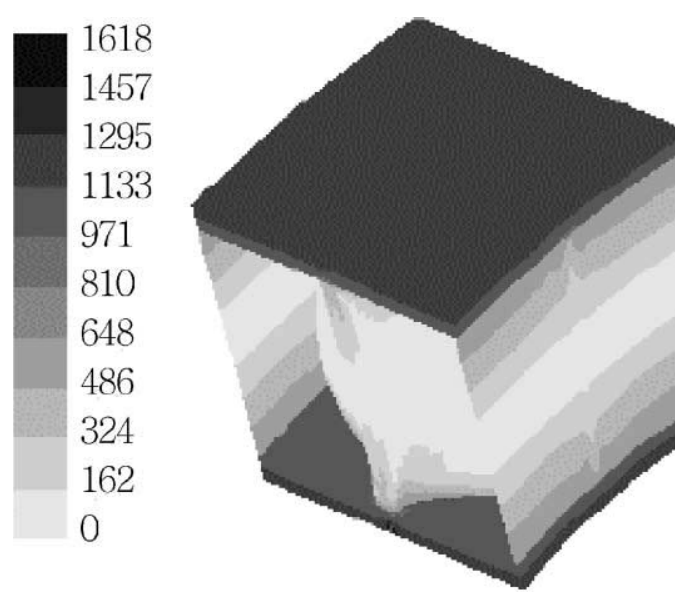

Fig. 10. Von Mises stress (MPa) contour lines on the deformed shape $\chi^{K_{11}}$.

\section{Behavior of the structures in transverse shear}

\subsection{Introduction}

Owing to what has been shown above, it can be stated that the homogenization method proves to be very accurate to provide the characteristics of an equivalent Kirchhoff Love plate. Nevertheless, it is not guaranteed that a Kirchhoff Love model is sufficient to describe the overall behavior of a sandwich panel. In order to investigate this question, let us consider a simple bending loading, still under the assumption of cylindrical bending.

The analysis is performed on a 10-period-long panel, in plane 13 , when condition $\vec{u}=\overrightarrow{0}$ is imposed at all the nodes in the cross-section of the origin, see Fig. 7(c). Force $F$ is taken such that $Q_{1}=10 \mathrm{E} 3 \mathrm{~N} / \mathrm{mm}$.

When the analysis of the heterogeneous panel is done, facings average deflection $w_{\mathrm{FE}}$ is determined and compared with that given by the analytical solution to the Kirchhoff Love plate problem, i.e.:

$w_{\mathrm{KL}}=-\frac{Q_{1}}{D_{1111}}\left(\frac{x_{1}^{3}}{6}-\ell \frac{x_{1}^{2}}{2}\right)$

The plot shown in Fig. 11(a) represents the results obtained for a panel with T2 stiffeners. One can notice a discrepancy between the two solutions. This discrepancy

Table 4

Effective moduli for the FRP honeycomb sandwich panel

\begin{tabular}{|c|c|c|c|c|c|c|c|c|}
\hline & \multicolumn{4}{|c|}{ Membrane behavior } & \multicolumn{4}{|c|}{ Bending behavior } \\
\hline & \multicolumn{3}{|c|}{ Moduli (GPa) } & \multirow[t]{2}{*}{ Poisson's ratio, $v_{12}^{m}$} & \multicolumn{3}{|c|}{ Moduli (GPa) } & \multirow[t]{2}{*}{ Poisson's ratio, $v_{12}^{f}$} \\
\hline & $\overline{E_{1}^{m}}$ & $E_{2}^{m}$ & $G_{12}^{m}$ & & $\overline{E_{1}^{f}}$ & $E_{2}^{f}$ & $G_{12}^{f}$ & \\
\hline Numerical homog. & 3.834 & 2.280 & 0.687 & 0.312 & 8.807 & 5.613 & 1.680 & 0.307 \\
\hline Ref. [18] & 3.813 & 2.206 & 0.648 & 0.303 & 8.777 & 5.537 & 1.627 & 0.301 \\
\hline
\end{tabular}



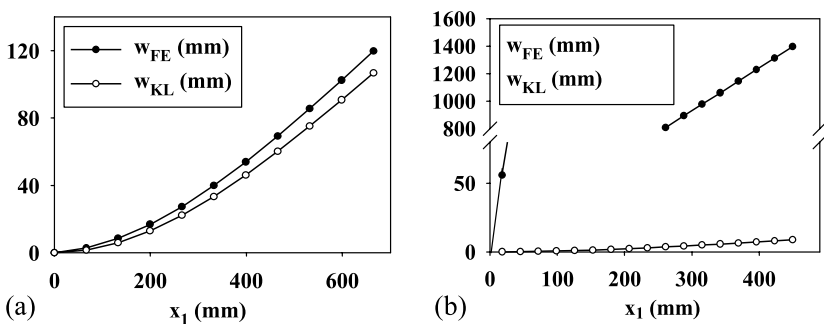

Fig. 11. Panel response in simple bending loadings (a) T2 stiffeners, (b) straight stiffeners.

is not significant for this type of stiffeners but is important for stiffeners that are straight (see Fig. 11(b)), curvilinear, or in hat shape, although all the structures studied have almost the same equivalent membrane and bending characteristics.

It appears that a Kirchhoff Love model is not completely satisfactory, and it is therefore necessary to take into account the transverse shear effects. As has been seen in the last results, these characteristics seem to be very sensitive to the core shape.

The problem is then to define an homogenization method for the transverse shear behavior.

Starting from 3D elastic problem, the asymptotic expansion method enables to recover, at the leading order, the Kirchhoff Love model. It can indeed be shown that the transverse shear forces associated with stresses $\sigma_{13}^{1}$ and $\sigma_{23}^{1}$ are zero. As a result, solving the cell problems described in Eq. (4) does not provide any information on the plates stiffness in transverse shear.

Refined plate theories, taking into account transverse shear effects, can be obtained using the asymptotic expansion method, including the higher-order terms of the expansion [26]. Such theories are complex, since they involved higher derivatives, and are therefore not used by engineers. This justifies attempts to derive an asymptotically correct Reissner Mindlin second order theory [27]. However, such a process isn't straightforward, because one has to choose the asymptotical correctness criterion. Moreover a higher-order microscopic problem needs to be solved. Consequently, this method will not be used in this paper.

Another way to proceed to obtain an equivalent Reissner Mindlin plate is to use the homogenization method proposed in [28]. Its starting point is the bidimensional equations of a Reissner Mindlin plate with periodic coefficients. As a consequence, the downside of this method is that the $3 \mathrm{D}$ behavior of the plate is only roughly taken into account, since it is a priori assumed that the heterogeneous 3D panel behaves like a heterogeneous Reissner Mindlin plate. The application field of this method is therefore limited to cells whose in-plane dimensions are very small compared to their thickness $[8,15,16]$, i.e. for $e \ll \varepsilon$, whereas for the example under consideration here, $e \approx \varepsilon$. It will be seen below that this method is inappropriate as far as the determination of the transverse shear stiffness is concerned.

It is then necessary to resort to another type of approach.

In the present work, we will use a numerical method to determine the corrugated core sandwich panel equivalent stiffness in transverse shear. Assuming the overall behavior being of Reissner Mindlin type, the transverse shear stiffness will be identified from the results obtained about a panel made of several cell periods. This is a difference from the method used for the homogenization of membrane and bending behaviors, where the finite element model only represented one period.

Let us mention that in the following, our study will be restricted to the corrugated sandwich panels of Fig. 1. However, the method proposed in this paper is also valid for the FRP honeycomb sandwich panel of Section 5 (in [18], its shown that a Timoshenko beam model is in good agreement with experimental data for three-point and four-point bendings).

\subsection{Principle of the method}

Let us re-consider the calculation made at the beginning of this section, assuming that the overall behavior in transverse shear and the membrane and bending behaviors are uncoupled, and that the associated stiffness matrix is diagonal, such that:

$\left\{\begin{array}{l}Q_{1} \\ Q_{2}\end{array}\right\}=\left[\begin{array}{cc}k_{1} & 0 \\ 0 & k_{2}\end{array}\right]\left\{\begin{array}{l}w_{, 1}+\theta_{2} \\ w_{, 2}-\theta_{1}\end{array}\right\}$

where $\theta_{1}$ and $\theta_{2}$ denote rotations about the $x_{1}$ and $x_{2}$ axes, respectively.

Then, the analytical solution to the equivalent homogeneous Reissner Mindlin plate problem is given by:

$w_{\mathrm{RM}}=-\frac{Q_{1}}{D_{1111}}\left(\frac{x_{1}^{3}}{6}-\ell \frac{x_{1}^{2}}{2}\right)+\frac{Q_{1} x_{1}}{k_{1}}=w_{\mathrm{KL}}+\frac{Q_{1} x_{1}}{k_{1}}$

One can further define from the finite element analysis of the heterogeneous panel:

$w_{\mathrm{FE}}^{*}\left(x_{1}\right)=w_{\mathrm{FE}}+\frac{Q_{1}}{D_{1111}}\left(\frac{x_{1}^{3}}{6}-\ell \frac{x_{1}^{2}}{2}\right)=w_{\mathrm{FE}}-w_{\mathrm{KL}}$

calculated with the value of $D_{1111}$ obtained from the homogenization method, and the accuracy of which has been established in Section 4.3. It is therefore assumed that Eq. (7) describes the membrane and bending behaviors of the equivalent Reissner Mindlin plate, when curvatures $\left\{K_{11}, K_{22}, 2 K_{12}\right\}$ are substituted with $\left\{\theta_{2,1},-\right.$ $\left.\theta_{1,2}, \theta_{2,2}-\theta_{1,1}\right\}$.

Then, if $w_{\mathrm{FE}}^{*}$ is plotted as a function of $x_{1}$, the graphs shown in Fig. 12(a) and (b) are obtained for T2 and straight stiffeners respectively:

A straight line is found in both cases, and this shows, from Eqs. (19) and (20), that the panel overall response 

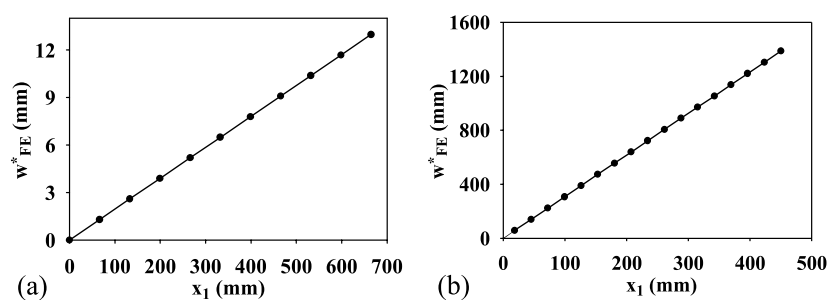

Fig. 12. Analysis of results in simple bending (a) T2 stiffeners, (b) straight stiffeners.

is similar to that predicted by a Reissner Mindlin model. It also provides a simple way to identify $k_{1}$ from the slope of the straight line using the least-square method.

However, one can notice that for T2 stiffeners, the difference between $w_{\mathrm{FE}}^{*}$ and $w_{\mathrm{FE}}$ is only about $10 \%$. Since $w_{\mathrm{FE}}^{*}$ is obtained from the homogenized bending characteristics of the panel, one may want to diminish the relative importance of $w_{\mathrm{KL}}$ in Eq. (20). Then, the length of the structure will be decreased so that $w_{\mathrm{FE}}^{*}$ is at least $20 \%$ lower than $w_{\mathrm{FE}}$ at the loaded tip of the panel.

For the study in plane 13, this has led us to generally consider 5-period-long panels, except for the T1 panel, where 3 periods are taken.

In plane 23 , the same procedure applies, and according to the same criterion, 5-period-long panels are considered, except for $\mathrm{T} 1$ and $\mathrm{H} 2$ panels, where 4 periods are studied. The boundary condition $\vec{u}=\overrightarrow{0}$ is imposed at all the nodes in the cross-section of the origin (core and facings). One finally uses the expression:

$w_{\mathrm{FE}}^{*}\left(x_{2}\right)=w_{\mathrm{FE}}+\frac{Q_{2}}{D_{2222}}\left(\frac{x_{2}^{3}}{6}-\ell \frac{x_{2}^{2}}{2}\right)=w_{\mathrm{FE}}+w_{\mathrm{KL}}$

knowing that the analytical solution $w^{*}$ is given by:

$w^{*}=\frac{Q_{2} x_{2}}{k_{2}}$

\subsection{Results}

For all the panels studied here, straight lines very well approximate the curves $w_{\mathrm{FE}}^{*}\left(x_{1}\right)$ in plane 13 and $w_{\mathrm{FE}}^{*}\left(x_{2}\right)$ in plane 23 obtained from the method described above. In turn, the values of $k_{1}$ and $k_{2}$ can be calculated. From these values, and using reference thickness $h_{\text {ref }}=5 \mathrm{~mm}$, we may define engineering constants in the form of shear moduli $G_{13}$ and $G_{23}$ such that:

$k_{1}=\frac{5}{6} h_{\mathrm{ref}} G_{13} ; \quad k_{2}=\frac{5}{6} h_{\mathrm{ref}} G_{23}$

The values calculated from Eq. (23) are gathered in Table 5.

Let us note that if the homogenization method [28] mentioned at the beginning of Section 6 is used (see the corresponding analytical expressions given in Appendix
Table 5

Panels homogenized characteristics in transverse shear

\begin{tabular}{lll}
\hline \multirow{2}{*}{$\begin{array}{l}\text { Structure (type of } \\
\text { stiffener) }\end{array}$} & \multicolumn{2}{c}{ Equivalent shear moduli $(\mathrm{GPa})$} \\
\cline { 2 - 3 } & $G_{13}$ & $G_{23}$ \\
\hline $\mathrm{S}$ & 0.207 & 240 \\
$\mathrm{H} 1$ & 22.3 & 149 \\
$\mathrm{H} 2$ & 1.58 & 139 \\
$\mathrm{~T} 1$ & 164 & 91.4 \\
$\mathrm{~T} 2$ & 123 & 164 \\
$\mathrm{C}$ & 0.400 & 95.3 \\
\hline
\end{tabular}

Moduli $G_{13}$ and $G_{23}$ for a $5 \mathrm{~mm}$ thick equivalent homogeneous plate.

A) one gets $G_{13}=0.792 \mathrm{GPa}$ and $G_{23}=0.974 \mathrm{GPa}$ for straight stiffener. These values are very far from the reference values obtained from the finite element analyses. Thus, such a homogenization method should not be applied to the type of panels considered here.

The values in Table 5 are to be compared with the shear modulus of steel $G=0.808 \mathrm{GPa}$, this value being a first approximation of the stiffened panel equivalent moduli in planes 13 and 23. Indeed, it can be stated that the stiffness in transverse shear of the stiffened panel is at least that of the underlying steel plate.

\subsection{Results analysis}

Compared to the case of the membrane and bending equivalent characteristics, significant differences can be noted.

First of all, the value of $G_{13}$ is very different from one panel to the other, and can be up to 1000 times lower than that of the reference stiffened panel. The results in plane 23 are less scattered, and the stiffness of the sandwich panels is on the same order of magnitude as that of the stiffened panel, although lower.

As a result, the alveolar panels have a strong anisotropic behavior in transverse shear, except for panels with triangular-shaped stiffeners. It appears then that the core shape has a strong influence on the value of modulus $G_{13}$, and that $\mathrm{T} 2$ triangular stiffeners at $60^{\circ}$ give the best transverse shear stiffness for sandwich panels. Finally, the values obtained show that it is necessary to use a Reissner Mindlin plate model, even for the stiffest panel (see Fig. 11(a)).

\section{Conclusions}

In this work, we have applied a periodic homogenization method to determine the equivalent membrane and pure bending characteristics of periodic plates. This method requires a few developments in a finite element package, and is then very easy to implement. As demonstrated in Section 4, this method yields very good results. Moreover, this method can be applied to many 
engineering structures, such as composite structures, see Section 5 .

However, the method does not make possible the evaluation of the transverse shear stiffness of sandwich panels. Therefore, we used another approach based on calculations in cylindrical bending. Then, after verifying that the panel overall response was in agreement with a Reissner Mindlin model, its transverse shear stiffness was identified from the results of the calculations.

This study allows to compare different types of alveolar structures with traditional stiffened structures, with a view to their applications in shipbuilding. Several conclusions can be drawn from the present work.

For a given mass per unit area, alveolar panels present a better overall bending stiffness than traditional stiffened panels. On the opposite, effective membrane stiffnesses are similar for all the panels. As a result, a weight reduction is possible, especially as far as the bending characteristics are concerned.

For the overall membrane and pure bending behaviors, the two driving parameters are the thickness of the facings and their spacing, whereas stiffeners geometry has only a minor influence. However, the equivalent behavior in transverse shear is very dependent on the stiffeners geometry, and it appears that triangular stiffeners have the best characteristics.

Finally, the important role played by shear stress in the behavior of such sandwich structures was highlighted.

Thanks to the methods presented in this work, we can determine the panels' effective characteristics according to a Reissner Mindlin plate. This will make it possible to model the alveolar panels as equivalent homogeneous plates, and will reduce the computation time when the finite element analysis is made on the whole ship.

\section{Acknowledgement}

The authors wish to thank Dr S. Bourgeois (L.M.A., Marseille, France) for the fruitful discussions they had with him on this subject.

\section{Appendix A}

According to [8], here are reported the analytical expressions for the homogenized characteristics of a panel with straight stiffeners (see Fig. 4(a)). It is recalled that the membrane bending coupling matrix $[B]$ is zero, that matrices $[A]$ and $[D]$ are symmetric, and that their terms with indices $\alpha \beta \gamma \delta$ are zero if index 1 or 2 appears an odd number of times.

By noting, $\gamma_{1}=t_{\mathrm{c}} / p$ and $\gamma_{2}=1-\gamma_{1}$, one gets, for membrane stiffnesses:

$$
\begin{aligned}
& \left.A_{1111}=\frac{\gamma_{1}}{t}+\frac{\gamma_{f}}{2 t_{\mathrm{f}}}\right)^{-1} \frac{E}{1-v^{2}} \\
& A_{1122}=v A_{1111} \\
& A_{2222}=\left(\gamma_{1} t+2 \gamma_{2} t_{\mathrm{f}}\right) E+v^{2} A_{1111} \\
& A_{1212}=\left(\frac{\gamma_{1}}{t}+\frac{\gamma_{2}}{2 t_{\mathrm{f}}}\right)^{-1} \frac{E}{2(1+v)}
\end{aligned}
$$

and for bending stiffnesses

$$
\begin{aligned}
& \left.D_{1111}=\frac{2}{3} \quad \frac{8 \gamma_{1}}{t^{3}}+\frac{8 \gamma_{2}}{t^{3}-\left(t-2 t_{\mathrm{f}}\right)^{3}}\right) \frac{E}{1-v^{2}} \\
& D_{1122}=v D_{1111} \\
& \left.D_{2222}=\frac{2}{3} \quad \frac{h^{3}}{8}-\gamma_{2}\left(\frac{h}{2}-e_{p}\right)^{3}\right) E+v^{2} D_{1111} \\
& \left.D_{1212}=\frac{2}{3} \quad \frac{\gamma_{1} t^{3}}{8}+\gamma_{2}\left(1-3 \zeta+3 \zeta^{2}\right) t_{\mathrm{f}}^{3}-\frac{\left(\frac{t}{2}-t_{\mathrm{f}}\right)^{6}}{T}\right)
\end{aligned}
$$

with

$$
\begin{aligned}
T & =p\left(\frac{\mu h^{3}}{8} \operatorname{coth}\left(\frac{2 \mu t_{\mathrm{c}}}{t}\right)+\mu^{\prime} e_{p}^{2} \operatorname{coth}\left(\frac{\mu^{\prime \prime}\left(p-t_{\mathrm{c}}\right)}{t_{\mathrm{f}}}\right)\right), \\
\zeta & =\frac{t}{2 t_{\mathrm{f}}}, \quad \mu=\frac{\sqrt{ } 3 k}{2}, \quad \mu^{\prime}=\left(1-3 \zeta+3 \zeta^{2}\right)^{1 / 2} \mu \\
\mu^{\prime \prime} & =\left(1-3 \zeta+3 \zeta^{2}\right)^{-1 / 2} \mu
\end{aligned}
$$

and where $k$ is the shape factor used for shear stiffness, taken here to be equal to $5 / 6$.

As for the equivalent stiffness in transverse shear obtained by homogenization of a Reissner Mindlin plate problem, the following holds, according to [8]:

$$
\begin{aligned}
& k_{1}=\frac{5}{6}\left(\frac{\gamma_{1}}{t}+\frac{\gamma_{2}}{2 t_{\mathrm{f}}}\right)^{-1} \frac{E}{2(1+v)} ; \\
& k_{2}=\frac{5}{6}\left(\gamma_{1} t+2 \gamma_{2} t_{\mathrm{f}}\right) \frac{E}{2(1+v)}
\end{aligned}
$$

\section{References}

[1] Noor AK, Burton WS, Bert CW. Computational models for sandwich panels and shells. Appl Mech Rev 1995;155(3):155 99.

[2] Triplett MH, Schonberg WP. Static and dynamic finite element analysis of honeycomb core sandwich structures. Struct Eng Mech 1998;6(1):95 113 .

[3] Burton WS, Noor AK. Assessment of continuum models for sandwich panel honeycomb cores. Comput Meth Appl Mech Eng 1997; 145(3 4):341 60 .

[4] Hohe J, Becker W. Effective stress strain relations for two dimensional cellular sandwich cores: Homogenization, material models, and properties. Appl Mech Rev 2002;55(1):61 87.

[5] Ko WL. Elastic constants for superplastically formed/diffusion bonded sandwich structures. AIAA J 1980;18(8):986 7.

[6] Nordstrand T, Carlsson LA, Allen HG. Transverse shear stiffness of structural core sandwich. Compos Struct 1994;27:317 29. 
[7] Caillerie D. Thin elastic and periodic plates. Math Meth Appl Sci 1984;6:159 91.

[8] Lewinski T, Telega JJ. Plates, laminates and shells. Asymptotic analysis and homogenization. Singapore: World Scientific; 1999.

[9] Bourgeois S, Cartraud P, Débordes O. Homogenization of periodic sandwiches. In: Vautrin A, editor. Mechanics of sand wich structures. Dordrecht: Kluwer; 1998. p. 1318.

[10] Bourgeois S. Modélisation numérique des panneaux structuraux légers. Ph.D. Thesis. University Aix Marseille II, 1997.

[11] Xu XF, Qiao P. Homogenized elastic properties of honeycomb sandwich with skin effect. Int J Solids Struct 2002;39:2153 88.

[12] Kohn RV, Vogelius M. A new model for thin plates with rapidly varying thickness. Int J Solids Struct 1984;20:333 50.

[13] Hohe J, Becker W. A refined analysis of the effective elasticity tensor for general cellular sandwich cores. Int J Solids Struct 2001;38:3689 717.

[14] Parton VZ, Kudryavtsev BA. Engineering mechanics of compos ite structures. Boca Raton, FL: CRC Press; 1993.

[15] Kalamkarov AL. Composite and reinforced elements of construc tion. New York: Wiley; 1992.

[16] Kalamkarov AL, Kolpakov AG. Analysis, design and optimiza tion of composite structures. Chichester: Wiley; 1997.

[17] Mouftakir L. Homogénéisation des structures ondulées. Ph.D. Thesis. University Metz, 1996.

[18] Davalos JF, Qiao P, Xu XF, Robinson J, Barth KE. Modeling and characterization of fiber reinforced plastic honeycomb sand wich panels for highway bridge applications. Compos Struct 2001;52:441 52 .
[19] Léné F. Contribution à l'étude des matériaux composites et de leur endommagement. Thesis. University Paris 6, 1984.

[20] Débordes O, Licht C, Marigo JJ, Mialon P, Michel JC, Suquet P. Calcul des charges limites de structures fortement hétérogènes. In: Grellier JP, editor. Tendances actuelles en calcul des structures. Paris: Pluralis; 1985. p. 5670.

[21] Devries F, Dumontet H, Duvaut G, Léné F. Homogenization and damage for composite structures. Int J Numer Meth Eng 1989; 27:285 98

[22] Guedes JM, Kikuchi N. Preprocessing and postprocessing for materials based on the homogenization method with adaptative finite element methods. Comput Meth Appl Mech Eng 1990;83: 14398.

[23] Michel JC, Moulinec H, Suquet P. Effective properties of composite materials with periodic microstructure: a computa tional approach. Comput Meth Appl Mech Eng 1999;172(1 4):109 43.

[24] Tsai SW. Theory of composites design. Dayton: Think Compos ites; 1992.

[25] Samtech. Samcef elements user manual, version 7.1, 1997.

[26] Goldenveizer AL, Kaplunov JD, Nolde EV. On Timoshenko Reissner type theories of plates and shells. Int J Solids Struct 1993;30(5):675 94.

[27] Sutyrin VG. Derivation of plate theory accounting asymptotically correct shear deformation. J Appl Mech 1997;64:905 15.

[28] Tadlaoui A, Tapiero R. Calcul par homogénéisation des micro contraintes dans une plaque hétérogène dans son épaisseur. J Méc Théor Appl 1988;7:573 95. 